\title{
Propuesta de un programa de prevención de lesiones en voleibol femenino infantil y cadete
}

\section{Proposal of a prevention's program of injuries in female volleyball infantil and cadete}

\author{
Óscar Del Castillo-Andrés*, Luis Toronjo-Hornillo, Inés Moya-Martínez y María del Carmen Campos-Mesa
}

${ }^{1}$ Departamento de Educación Física y Deporte, Universidad de Sevilla (España).

\begin{abstract}
Resumen: El objetivo de este estudio es analizar la respuesta motriz de dos grupos de jugadoras de voleibol de enseńanza secundaria obligatoria, ante una caída no intencionada de espalda y determinar qué efectos de protección se observan en la caída tras la participación en el programa sobre formas seguras y protegidas de caer Safe Fall-Safe Schools ${ }^{\odot}$.

Aplicamos una investigación cuasi-experimental, a 27 jugadoras, de edades entre los 13 y los 16 ańos, que practican como actividad extraescolar voleibol en el I.E.S. Ramón y Cajal de Tocina, Sevilla, España. Los datos se recogieron antes y después de la intervención mediante la escala de observación ad hoc INFOSECA. Se aplicó estadística descriptiva y correlacional. Se consideró la significatividad estadística en $p<0.05$ (prueba de McNemar). Los resultados señalan que es posible asimilar con éxito los movimientos asociados a una caída hacia atrás para aumentar la protección y seguridad de las menores deportistas.

Palabras clave: Safe Fall-Safe Schools; caídas; salud; innovación educativa; voleibol.
\end{abstract}

Abstract: The aim of this study is to analyze the motor response of two groups of volleyball players of compulsory secondary education, before an unintentional fall of the back, and to determine what effects are observed in the fall after participating in the program Safe Fall-Safe Schools@.

A quasi-experimental research has been applied to 27 players, between the ages of 13 and 16, who practice as an extra-curricular volleyball activity in the I.E.S. Ramón y Cajal de Tocina, Seville, Spain. The data was collected before and after the intervention, using the INFOSECA ad hoc observation scale. Descriptive and correlational statistics were applied. The criterion to consider the statistical significance was $p<0.05$ (McNemar test).

The results obtained indicate that through the implementation of the program it is possible to assimilate successfully the technical movements associated with a fall backwards that contribute to increase the protection and safety of the minors athletes.

Keywords: Safe Fall-Safe Schools; falls; health; educational innovation; volleyball.

\section{Introducción}

Según los datos aportados en el último informe de la OMS y UNICEF (diciembre de 2018), cada día fallecen más de 2000 menores como consecuencia directa de las lesiones no intencionales (accidentales). En esta línea, decenas de millones de niños son hospitalizados con lesiones que frecuentemente les provocan discapacidad permanente. En Europa los fallecimientos por lesiones no intencionadas constituyen la primera causa de muerte en la infancia entre los 5 y 18 ańos; y sólo en España, llegaron a fallecieron 149 niños menores de 15 años en 2014 (Esparza y Mintegui, 2016).

El coste sanitario que suponen los accidentes es muy elevado, debido a que el $35 \%$ de los accidentados acuden al servicio de urgencias de pediatría de los hospitales, teniendo que ser ingresados el 13\% de ellos (Concheiro et al., 2006).

La Alianza para la Seguridad Infantil Europea (European Child Safety Alliance), es un organismo nacido de una iniciativa de la Asociación Europea para la Seguridad del con-

Dirección para correspondencia [Correspondence address]: Óscar Del Castillo-Andrés. C/ Pirotecnia s/n, 41013, Sevilla (España). (https://orcid. org/0000-0002-3024-1097). E-mail: ocastillo@us.es sumidor, cuyo objetivo es prevenir las lesiones infantiles en Europa a través de diferentes programas:

- Plan de acción para la seguridad infantil (CSAP).

- Herramienta para abordar el trauma de la niñez, las lesiones y la seguridad infantil (TACICS).

- Acción conjunta sobre el control de las lesiones en Europa (JAIME).

Los miembros que integran la alianza realizan un informe sobre la seguridad infantil en cada país, para cada uno de los programas. España participa en los tres programas y ha realizado la recogida de datos sobre las lesiones infantiles a través del Instituto Nacional de Estadística, la Encuesta Nacional de salud, la Detección de Accidentes Domésticos y de Ocio (D.A.D.O.), los Informes de siniestralidad de la Dirección General de Tráfico, el Observatorio Toxicológico (perteneciente a la Sociedad Española de Urgencias Pediátricas), así como a través de iniciativas privadas (Esparza y Mintegui, 2016).

Según la Encuesta Nacional de Salud (2012) los lugares más frecuentes donde tienen lugar los accidentes infantiles son, de mayor a menor: el hogar, la vía pública, el colegio o 
lugar de estudio y las instalaciones deportivas. Las lesiones más comunes registradas en los servicios de urgencias son los traumatismos cráneo-encefálico, las fracturas de extremidades, los politraumatismos, las contusiones abdominales y las heridas en las extremidades (Concheiro et al., 2006). Así mismo, las caídas, suponen la tercera causa de muerte por lesión no intencionada en la Unión Europea (UE) en menores de 19 años y la primera causa de ingreso por lesión accidental (Esparza y Mintegui, 2016). En Andalucía, el 4,3\% de las muertes sufridas por menores de 15 años son ocasionadas por caídas (Junta de Andalucía, 2016).

Ante este importante problema de salud pública se hace necesario el trabajo de investigación y el desarrollo de programas orientados a reducir el riesgo de sufrir un accidente o lesión. En la búsqueda de este objetivo se han elaborado programas por entidades como la Fundación Mapfre, que ha editado la "Guía para padres sobre la prevención de lesiones no intencionadas en la edad infantil" de Esparza y Mintegui (2016), la "Guía de prevención de accidentes infantiles" de Soriano (2008), o a través del programa de prevención de accidentes "Aprende a crecer con seguridad", de la Junta de Andalucía (2016).

Más allá de los programas de prevención que se proponen desde organismos e instituciones, como las citadas anteriormente (todas ellas centradas en el estudio de la biomecánica de la caída o en investigaciones sobre estrategias de formas de caer que reduzcan las lesiones en una caída), no hemos encontrado programas que de forma específica tenga como objetivo la implementación de un método de enseñanza sobre las caídas en la población de menores en edad escolar, que contribuya a la disminución de las consecuencias lesivas de las caídas accidentales. En este sentido, algunos estudios sobre el programa "Safe Fall-Safe Schools ${ }^{\Theta}$ " (Toronjo, Del Castillo, Campos, Díaz y Zagalaz, 2018), presentan la posibilidad de enseñar a caer de una forma segura mediante la implementación de este programa, el cual se centra en los fundamentos básicos del judo, de forma adaptada a los grupos con los que se trabaja y mediante el trabajo de desplazamientos, equilibrio, habilidades motoras básicas y específicas, con el fin de automatizar los movimientos para garantizar que los niños se protejan en caso de una caída involuntaria hacia atrás.

En dicho programa, los ejercicios propuestos cumplen con los principios metodológicos de la enseñanza de actividades físicas y deportivas, estableciendo un aumento progresivo de la dificultad mediante diferentes niveles y teniendo en cuenta las siguientes variables: altura inicial y máxima del centro de gravedad con respecto a la superficie final del contacto, número de ejes implicados en el movimiento global, número de participantes implicados en el ejercicio, complejidad en la toma de decisiones y la inercia generada y velocidad de los movimientos (Del Castillo et al. 2017).

Estos indicadores de las caídas, se encuentran en algunas actividades deportivas como el voleibol y el judo. En voleibol los participantes en la recepción media y baja de balones lejanos, han de lanzarse para golpear la pelota, realizando una "caída de voleibol" en la dirección del desplazamiento, en la que realizan un movimiento circular durante la caída para retomar la postura de pie. En judo es un deporte en el cual el objetivo consiste en proyectar al oponente sobre el tatami, consiguiendo los judocas reducir la energía del impacto mediante el golpeo del suelo con la mayor superficie posible o rodando sobre el cuerpo.

En una revisión sistemática sobre formas seguras de caer realizada por Moon y Sosnoff (2017) se concluyen, como estrategias para afrontar las caídas de forma segura, la flexión de las piernas, flexión de los codos, rodar hacia delante, balanceo hacia atrás en las artes marciales, golpeo del suelo en las artes marciales, musculatura relajada y escalonamiento. Todos los sistemas estudiados excepto el golpeo del suelo utilizado en artes marciales, contribuyeron a reducir el impacto del cuerpo contra el suelo durante las caídas, por lo que podríamos afirmar que enseñar técnicas para caer de forma más segura a la población de menores en edad escolar, puede contribuir a reducir el impacto sobre el cuerpo provocado en una en caída.

En función de lo anteriormente expuesto, el objetivo de la siguiente investigación es constatar si la participación de las jugadoras en el programa Safe Fall-Safe Schools ${ }^{\odot}$ permite asimilar gestos motrices de protección ante una caída sobrevenida en jugadoras de escuelas deportivas (nivel 1) y de los equipos federados (nivel 2) de voleibol.

\section{Material y Método}

\section{Muestra}

La muestra sobre la que se efectúa el estudio se compone de un total de 27 alumnas de educación secundaria, del I.E.S. Ramón y Cajal de Tocina, Sevilla, España. Practican como actividad extraescolar voleibol y pertenecen a las categorías infantil y cadete. La edad media está comprendida entre los 12 y los 16 ańos $(M=14.1$ ańos, $D T=2.45)$.

Las jugadoras compiten en dos niveles: unas en la liga municipal (nivel 1) y el resto en competición federada (nivel 2). Las de primer nivel cuentan con uno o dos años de práctica y las segundas más de tres años, lo que supone en este grupo una exigencia superior en cuanto a táctica y técnica.

\section{Procedimiento}

En primer lugar, se informó detalladamente a las participantes en el estudio y a sus padres y madres, de la intervención a desarrollar, explicándose el programa Safe Fall-Safe Schools ${ }^{\circledR}$, el objetivo del estudio y el procedimiento a seguir. 
Se obtuvo el consentimiento informado de los tutores de las menores.

El período de implementación fue de cuatro semanas, más una sesión anterior en la que se hizo el pre-test y otra posterior para el pos-test. Como implementación, se desarrollaron doce sesiones basadas en el empleo de fundamentos y elementos técnicos del Safe Fall-Safe Schools ${ }^{\odot}$, contextualizados a los requerimientos de la etapa educativa a la que pertenecen las jugadoras. Se llevaron a cabo ejercicios de nivel 1 durante la mayoría de las sesiones y se finalizó con algunos de nivel 2 . Las doce sesiones que se realizaron tuvieron una duración de 20 a 25 minutos cada una y se impartían al comienzo de los entrenamientos diarios de las deportistas. En estas sesiones se desarrollaron ejercicios de los niveles 1 y 2 del programa Safe Fall-Safe Schools ${ }^{\circledR}$.

Tras la implementación se volvió a evaluar a las jugadoras mediante la escala de observación "INFOSECA", recogiéndose las respuestas motrices realizadas tras haber participado en el programa.

\section{Instrumento}

La evaluación de la caída hacia atrás se realiza mediante la escala de observación sistemática INFOSECA, que valora cinco gestos de protección asociados a una caída hacia atrás: la flexión o no del cuello por parte del alumnado, el apoyo de las manos en el suelo, si se consigue rodar sobre la espalda y el mantenimiento de rodillas y caderas flexionadas durante la caída.

\section{Resultados}

La tabla 1 refleja los resultados obtenidos en el pre-test y el post-test realizados a las jugadoras con respecto a la variable de la flexión del cuello. Los datos anteriores a la implementación del programa, recogen un porcentaje medio de jugadoras que flexionaban el cuello, llevando la barbilla al pecho con la intención de proteger la cabeza como gesto de protección, pasando ser la totalidad tras participar en el programa.

Tabla 1. Resultados Pre-test y Pos-test programa Safe Fall-Safe Schools ${ }^{\odot}$.

\begin{tabular}{lcccccccc}
\hline & \multicolumn{3}{c}{ Pre-test } & \multicolumn{3}{c}{ Pos-test } \\
\hline & Sí & $\%$ & No & $\%$ & Sí & $\%$ & No & $\%$ \\
\hline Cuello & 11 & 40,7 & 16 & 59,5 & 27 & 100 & 0 & 0 \\
Manos & 18 & 66,7 & 9 & 33,3 & 27 & 100 & 0 & 0 \\
Tronco & 12 & 44,4 & 15 & 55,6 & 27 & 100 & 0 & 0 \\
Cadera & 20 & 74,1 & 7 & 25,9 & 27 & 100 & 0 & 0 \\
Rodillas & 22 & 81,5 & 5 & 18,6 & 27 & 100 & 0 & 0 \\
\hline
\end{tabular}

Con respecto al apoyo de las manos en el suelo en el momento del impacto (Tabla 1), obtenemos que el más de la mitad de las jugadoras no lo hicieron en pre-test. La mayoría optaron por dejar los brazos estirados por encima de la línea horizontal del cuerpo durante la caída. En el test realizado tras las sesiones ninguna apoyó las manos en el suelo.

La variable tronco (Tabla 1), en la que se debería realizar un balanceo sobre la espalda, muestra que casi la mitad del grupo realiza correctamente este movimiento circular sobre la espalda. En el pos test todas las jugadoras realizan correctamente el movimiento.

Los elementos evaluados de la flexión de cadera y rodillas, se muestran en la Tabla 1, son los más elevados obtenidos en el pre-test, alcanzando unos porcentajes cercanos y por encima del 75\% respectivamente. Al igual que el resto de vari- ables evaluadas durante la caída, el total de la muestra realiza correctamente la flexión de caderas y rodillas tras participar en el programa.

Las estadísticas de contraste de significado exacto bilateral aplicadas en los test realizados anteriormente y con posterioridad a participar en el programa de caídas, han puesto de manifiesto la existencia de diferencias significativas.

Se aplicó la prueba de McNemar $(p<0.05)$ en las 5 variables evaluables de la caída. En la Tabla 2 podemos ver que la prueba presentó un significado exacto (bilateral) por debajo de 0.05 en todas las variables, excepto en la variable rodillas, dato asociado a que el $81,5 \%$ de las chicas participantes en el programa ya flexionaban las rodillas a la hora de realizar una caída hacia atrás.

Tabla 2. Resultados significación exacta (bilateral) del programa Safe Fall-Safe Schools ${ }^{\odot}$.

\begin{tabular}{cccccc}
\hline $\begin{array}{c}\text { Significación } \\
\text { exacta (bilateral) }\end{array}$ & Cuello & Manos & Tronco & Cadera & Rodillas \\
\cline { 2 - 6 } & $0,000^{\mathrm{b}}$ & $0,004^{\mathrm{b}}$ & $0,000^{\mathrm{b}}$ & $0,016^{\mathrm{b}}$ & $0,063^{\mathrm{b}}$ \\
\hline
\end{tabular}


Comparación entre los niveles de las jugadoras

La comparación de los datos obtenidos según los niveles de las deportistas está recogida en la Tabla 3, donde se reúnen los datos registrados para los niveles 1 y 2 , del pre test y del post-test.

Tabla 3. Datos obtenidos de los niveles 1 y 2 de las deportistas en el pre-test y pos-test.

\begin{tabular}{|c|c|c|c|c|c|c|c|c|}
\hline & \multicolumn{4}{|c|}{ PRE TEST } & \multicolumn{4}{|c|}{ POS TEST } \\
\hline & \multicolumn{2}{|c|}{ Nivel 1} & \multicolumn{2}{|c|}{ Nivel 2} & \multicolumn{2}{|c|}{ Nivel 1} & \multicolumn{2}{|c|}{ Nivel 2} \\
\hline & Sí & $\%$ & Sí & $\%$ & Sí & $\%$ & SÍ & $\%$ \\
\hline Cuello & 3 & 33,3 & 8 & 44,4 & 9 & 100 & 18 & 100 \\
\hline Manos & 4 & 44,4 & 14 & 77,8 & 9 & 100 & 18 & 100 \\
\hline Tronco & 0 & 0 & 12 & 66,7 & 9 & 100 & 18 & 100 \\
\hline Cadera & 4 & 44,4 & 16 & 88,9 & 9 & 100 & 18 & 100 \\
\hline Rodillas & 7 & 77,8 & 15 & 83,3 & 9 & 100 & 18 & 100 \\
\hline
\end{tabular}

\section{Discusión}

Tras la implementación del programa de caídas Safe Fall-Safe Schools ${ }^{\oplus}$ y la realización de varias sesiones basadas en los fundamentos técnicos básicos del judo sobre las caídas, adaptados al nivel de la muestra, todas las participantes han ejecutado las respuestas motrices que se relacionan con el objetivo de contribuir a reducir las posibles lesiones, o la gravedad de las mismas, que se pueden producir por una caída hacia atrás.

Los movimientos técnicos que se proponen en el programa Safe Fall-Safe Schools ${ }^{\circledR}$ están de acuerdo los estudios de Jaskólski y Nowacki (1972), en los cuales se plantea para reducir la energía del impacto en la caída, que la superficie de contacto del cuerpo al tocar con el suelo realice un movimiento circular que aumente la superficie de contacto, al mismo tiempo que disminuye el tiempo de contacto de cada punto con el suelo. Esto coincide con el movimiento de balanceo del tronco, que debe tocar el suelo con la parte baja de la espalda, iniciando un movimiento circular hacia la parte superior de la espalda, sin llegar nunca a tocar o apoyar la cabeza en el suelo.

En la revisión sistemática de formas seguras de caer llevada a cabo por Moon y Sosnoff (2017) también se encuentran propuestas técnicas similares a las realizados en el programa Safe Fall-Safe Schools ${ }^{\odot}$. En esta revisión se reconocieron siete maniobras que contribuyen a mejorar la seguridad en las caídas, relacionándose con las caídas hacia atrás la flexión de las rodillas (sentadilla) y el balanceo del tronco. Los movimientos técnicos presentes en el programa Safe Fall-Safe Schools ${ }^{\odot}$ para realizar la caída hacia atrás de forma segura, pasan por flexionar la cadera y las rodillas, es decir, se debe realizar una sentadilla y describir un movimiento circular o balanceo con la espalda como hemos nombrado anteriormente evitando que la cabeza golpee el suelo.
La comparación de los datos de este trabajo y del estudio elaborado por Toronjo et al. (2018) sobre alumnado de educación secundaria, presentan resultados semejantes tras la aplicación del programa, es decir en el pos-test, donde casi la totalidad de los participantes responden con la realización de los movimientos necesarios para realizar una caída segura. En cuanto a los datos obtenidos antes de participar en el programa si existen importantes diferencias, pues no más del $15 \%$ del alumnado de dicho estudio realiza correctamente las variables analizadas, mientras que el porcentaje más bajo encontrado en nuestro análisis es del $40.7 \%$ para la variable de flexionar el cuello antes del iniciar el programa.

También los datos obtenidos por parte de las jugadoras de nivel 2 con respecto a los movimientos realizados de forma correcta en el pre-test, son más elevados que las de nivel 1. Es probable que estas diferencias estén relacionadas con la enseñanza a las jugadoras federadas de las caídas propias del deporte de voleibol, que comparten elementos técnicos como los desarrollados en el Safe Fall-Safe Schools ${ }^{\odot}$, como son flexión de cadera y rodillas y el balanceo sobre la espalda.

En esta dirección de evaluar la respuesta motriz a las caídas por practicantes de deportes diferentes Mroczkowski y Mateusz (2015) llevaron a cabo un estudio en el que se realizaron varias pruebas a estudiantes que asistían a clases de educación física y en algunos casos hacían deportes en sus actividades extraescolares, para determinar si la práctica deportiva facilitaba la mejora de las cualidades de coordinación y equilibrio necesarias para reducir el riesgo de sufrir una lesión ante una caída. Los resultados obtenidos confirmaron que la práctica de actividad física o de algún deporte no reducía la posibilidad de padecer una lesión en una caída. Pero se dio la excepción en uno de los participantes, que practicaba judo y realizó las pruebas de forma excelente. De esta forma se podría considerar que la enseñanza algunos deportes en los que 
se incluye la enseńanza y entrenamiento de técnicas de caída, como es el caso del judo o como ha sucedido en este estudio con la caída de voleibol, podrían reducir los riesgos de sufrir una lesión en una caída, pues ambos mejoraban los resultados de las investigaciones llevadas a cabo.

Entre las limitaciones del estudio está la escasa bibliografía encontrada sobre programas llevados a cabo para intentar reducir o evitar lesiones durante las caídas, debido a lo cual no se ha podido realizar el contraste de los resultados de este estudio con otros programas. Igualmente, el programa Safe Fall-Safe Schools ${ }^{\Theta}$ solamente se ha implementado con anterioridad de forma generalizada en centros escolares de primaria y secundaria, con alumnos que practicaban mayoritariamente como actividad física las clases de educación física, por lo que no ha sido aplicado con anterioridad, con deportistas que entrenen casi a diario.

También cabe reseñar que únicamente se ha realizado sobre jugadoras de voleibol sin contar con participantes masculinos, debido a que en la localidad en la que se ha llevado a cabo el estudio, no existe ningún equipo masculino de voleibol, ni de escuelas deportivas ni federado.

En relación con su aplicación al deporte del voleibol, muchas de las caídas se producen hacia los lados y hacia delante, al intentar los deportistas llegar a los balones, aunque estén lejos de su alcance, por lo que se podrían ampliar los ejercicios para aprender a realizar con seguridad caídas en otras direcciones como hacia delante y laterales, lo que requeriría también de la creación de nuevas escalas de observación para estas caídas.

\section{Conclusiones}

La puesta en marcha de esta intervención de formas seguras y protegidas de caer mediante el programa Safe Fall-Safe Schools ${ }^{\odot}$, permite la asimilación de los movimientos técnicos necesarios para realizar una caída hacía atrás de forma segura. Esto podría contribuir a la reducción del número de lesiones o de la gravedad de las mismas, ante una caída sobrevenida durante la práctica deportiva.

El programa Safe Fall-Safe Schools ${ }^{\circledR}$ es una propuesta que se adecúa a las peticiones de la Organización Mundial de la Salud (OMS), que señala como objetivo importante la implementación y puesta en marcha de programas educativos que lleguen a reducir la siniestralidad infantil ocasionada por caídas.

En España podría incorporarse como una herramienta útil en los centros educativos y pasar a formar parte del currículum de la asignatura de educación física ya que en la legislación educativa actualmente vigente (Reales Decretos 126/2014 y 1105/2014), está presente de forma específica el judo dentro del contenido curricular.

\section{Referencias}

1. Arbós, J., Rovira, M., Llobera, J. y Bonet, M. (1995). Accidentes infantiles en atención primaria. Revista española de salud pública, 69 (1), 97-103.

2. Calero, M., Vives, M., García, M., Soriano, M., Calero, M. y Berral, E. (2008). Impacto de una campańa escolar de prevención de riesgos. Revista de Psicología del Trabajo y de las Organizaciones, 24(2), 169-186.

3. Campos, M.C., Corral, J., Chacón, F. y Castañeda, C. (2017). Need to introduce teaching of safe and protected teacher training techniques (Safe Fall). Journal of Sport and Health Research, 9(1), 115-120.

4. Campos, M.C., Del Castillo, O., Castañeda, C. y Toronjo, L. (2015). EDUCAJUDO: innovación educativa y de salud en mayores. Aprendiendo a caerse (yoko-ukemi) Revista Iberoamericana de Ciencias de la Actividad Física y el Deporte, 4(3), 1-10.

5. Concheiro, A., Luaces, C., Quintillá, J.M., Delgado, L. y Pou, J. (2006). Accidentes infantiles: diseño y aplicación de un registro hospitalario del niño accidentado. Barcelona: Servicio de pediatría. Sección de urgencias Hospital Sant Joan de Déu, 18, 275-281.

6. DeGoede, K.M., Ashton-Miler, J.A. y Schultz, A.B. (2003). Fall-related upper body injuries in the older adult: a review of the biomechanical issues. Journal of biomechanics, 36(10), 43-53.

7. Del Castillo, O., Toronjo, L., González, G. y Toronjo, M.T. (2017). Propuesta de intervención "Saffe Fall". Prevención de lesiones en escolares mediante formas seguras de caer. Journal of sport and Health Research, 9(1), 137-142.

8. Esparza, M.J. (2009). Prevención de lesiones infantiles por accidentes. Revista pediátrica Atención Primaria, 11(6), 57-66.

9. Esparza, M. y Mintegui, S. (2016). Guía para padres sobre la prevención de lesiones no intencionadas en la edad infantil. Madrid: Asociación Es- pañola de Pediatría. Fundación Mapfre.

10. Jagiello, W., Wójcicki, Z. y Barczynski, B. (2014). Optimal body balance disturbance tolerance skills as a meth-odological basics for selection of the firefighters to solve difficult tasks of rescue. Annals of Agricultural and Environmental Medicine, 21(1), 148-155.

11. Jaskólski, E. y Nowacki, Z. (1972). Teoria, metodyka i systematyka miękkiego padania. CzęśćI. Teoria miękkiego padania. Wroclaw:WSWF, $11,83-88$.

12. Junta de Andalucía (2016). Programa aprende a crecer con seguridad. Jaén: Consejería de Empleo.

13. Kalina, R.M. y Barczynski, B. (2010). Eko-Agro-Fitness continuous program of health-oriented and ecological education un the family, among friends or individually implemented. Archives of budo, 6(4), 179184.

14. Kalina, R.M. (2013). Non-apparatus safe falls preparations test (NASFPT). Archives of budo, 9(4), 255-265.

15. Kalina, R.M., Jagietto, W. y Barczynski, B.J. (2013). The method to evaluate the body balance disturbation tolerance skills. Archives of budo, 9(1), 59-69.

16. Latorre, P.A. y Pantoja, A. (2012). Diseño y validación de una escala de percepción del riesgo en actividades físico deportivas escolares. Retos. Nuevas tendencias en Educación Física, Deporte y Recreación, 21, 25-29.

17. Latorre, P.A., Cámara, J.C., Pantoja, A. y Izquierdo, T. (2013). Factores psicoeducativos que determinan la propensión al accidente deportivo en escolares: Diseño y validación de un cuestionario de evaluación. Anales De Psicología, 29(2), 462-469.

18. Luraschi, N., Franco, R. y Servin, G. (2003). Accidentes en la infancia y la adolescencia. Sociedad Paraguaya de Pediatría, 30(2), 17-20. 
19. Moony. P. y Sosnoff, J. J. (2017). Safe landing strategies during a fall: systematic review and meta-analysis. Archives of Physical Medicine and Rehabilitation, 98(7), 83-94.

20. Morrongiello, B.A. (1997). Children's perspectives on injury and close call experiences: Sex differences in injury-outcome processes. Journal of Pediatric Psychology, 22(4), 499-512.

21. Mroczkowski, A. y Mateusz, M. (2015). The susceptibility to body injuries during a fall and abilities related to motor coordination of children aged 10 to 12. Archives of Budo Science of Martial Arts and Extreme Sports, 11, 65-71.

22. Organización Mundial de la Salud (2018). Caídas. Recuperado de http://www.who.int/es/news-room/fact-sheets/detail/falls

23. Pascual, A. y Aragües, G. (1998). Lesiones deportivas y rasgos de ansiedad en los jugadores de fútbol. Medicina Clínica, 111(2), 45-48.

24. Peterson, L., Brazeal, T., Oliver, K. y Bull, C. (1997). Gender and developmental patterns of affect, belief and behaviors in simulated injury events. Journal of Applied Developmental Psychology, 18(4), 531-546.

25. Pocecco, E., Gatterer, H., Ruedl, G. y Burtscher, M. (2012). Specific exercise testing in judo athletes. Archive of Budo, 8, 133-139.

26. Pocecco, E., Ruedl, G., Stankovic, N., Sterkowicz, S., Del Vecchio, F.B. y Gutiérrez-García, C. (2013). Injuries in judo: a systematic literature review including suggestions for prevention. British Journal of Sport
Medicine, 47(11), 39-43.

27. Real Academia Espańola. (2014). Diccionario de la lengua española (23a ed.). Consultado en http://www.rae.es/

28. Schwebel, D. y Barton, B. (2005). Contributions of multiple risk factors to child injury. Journal of Pediatric Psychology, 30(7), 553-561.

29. Sinaki, M., Brey, R. H. y Larson, D. K. y Kaufman, K.R. (2005). Significant reduction in risk of falls and back pain in osteoporotic-kyphotic women through a spinal prorioceptive extension exercise dynamic (SPEED) program. Mayo Clinic Proceedings, 80, 849-855.

30. Soriano, M. (2008). Accidentes infantiles. Jaén: Junta de Andalucía Consejería de Empleo.

31. Sran, M.A., Stotz, P.J., Normandin, S.C. y Robinovitch, S.N. (2010) Age differences in energy absorption in the upper extremity during a descent movement: implications for arresting a fall. The Journals $O f$ Gerontology, 65, 312-317.

32. Tencer, A. F. (2005). Biomechanics of falling. Mayo Clinics, 80(7), 847848.

33. Toronjo, L., Del Castillo, Ó., Campos, M.C., Díaz, V.M. y Zagalaz, M.L. (2018). Effect of the Safe Fall Progamme on Children's health and safety: dealing proactively with backward falls in physical education classes. Sustainability, 10(4), 1-8. https://doi.org/10.3390/su10041168 